Lamia T Rejab

BDS, MSc (Asst. Prof.)

Rand Sh Al-Ani

BDS, MSc (Asst. Lec.)

\section{The Relation of Bruxism with Teeth Attri- tion and Condylar Asymmetry (Clinical and Radiological Assay)}

Department of Prosthetic Dentistry

College of Dentistry, University of Mosul

Department of Oral and maxillofacial Surgery

College of Dentistry, University of Mosul

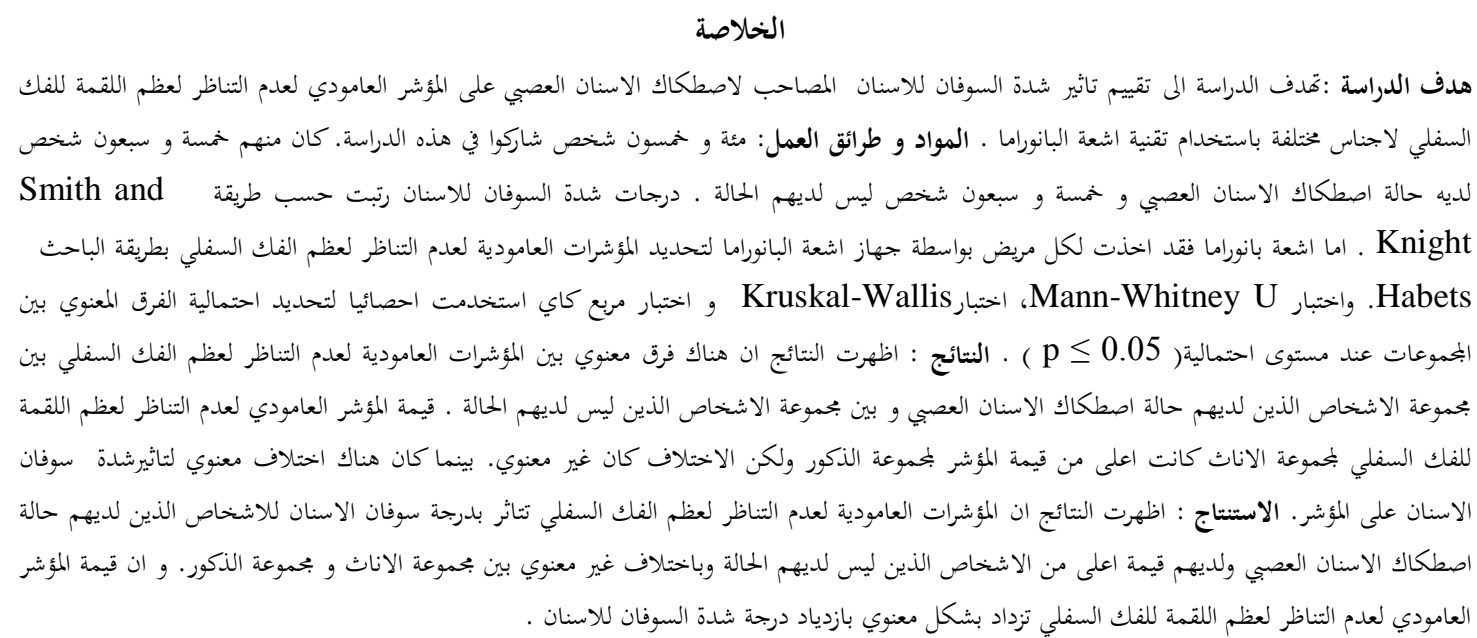

ABSTRACT

Aim : The paper aims to evaluate the effect of teeth wear severity associated with bruxism on the condylar height asymmetry index among different gender using panoramic radiograph viewing technique. Materials and methods: One hundred fifty subjects were participate in this study. Seventy five were bruxers and seventy five were non-bruxers. Degree of tooth wear severity was graded using Smith and Knight method. The panoramic radiographs were taken for each patient using a panoramic apparatus to determine vertical mandibular height asymmetry indices by a method developed by Habets et al. MannWhitney U test, Kruskal-Wallis test and Pearson Chi-Square test were used to determine the possible statistically significant differences between groups set at $(P \leq 0.05)$.Results: The results showed there are significant differences between the vertical mandibular height asymmetry indices of the bruxers and non-bruxers subjects. The female bruxer subjects had the higher condylar index value than that of the male but statistically, there is no significant difference, while the severity of tooth wear had a significant different affect on the condylar indices. Conclusions: The results showed that the vertical mandibular asymmetry indices were affected by the tooth wear associated with bruxer subjects. The bruxer subjects had the higher value than the non-buxer subjects and there is no significant difference of the condylar index of bruxer subjects among different genders. While, condylar index value increased significantly as the tooth wear severity grade increased.

Key word: Bruxism, tooth wear, condylar index, panoramic radiography.

Rejab LT, Al-Ani RSh. The Relation of Bruxism with Teeth Attrition and Condylar Asymmetry ( Clinical and Radiological Assay). Al-Rafidain Dent J. 2011; 11(2): 265-274.

Received: 21/2/2010 Sent to Referees: $28 / 2 / 2010$

Accepted for Publication: 25/3/2010

\section{INTRODUCTION}

Bruxism is a common parafunctional activity that includes grinding or clenching of the teeth. It may occur without any signs, symptoms, or harmful effect to the masticatory system but may become problematic by contributing to increased tooth wear and pain. ${ }^{(1)}$

Bruxism is considered the most detrimental among all the parafunctional activities of the stomatognathic system, being considered a risk factor for temporomandibular disorders, and in particular for myofascial pain. ${ }^{(2,3)}$ Bruxism was reported in 
20-25\% with a positive correlation between bruxism and clinical signs of dysfunction. ${ }^{(4)}$

Bruxism has been defined as nonfunctional (parafunctional) movements of the mandible, with or without audible sound occurring during the day or night. ${ }^{(5)}$ Basically, two groups of etiological factors in bruxism can be distinguished, peripheral (morphological) factors and central (pathophysiological and psychological) factors. ${ }^{(6,7)}$ The incidence of bruxism seems to vary according to the definition and diagnostic criteria used, the population sampled and the design of questionnaire employed. $^{(5)}$

Bruxism has been recognized to be one of the most destructive dental habits. This destruction may determine an impairment of the quality of life and also reflect a major masticatory dysfunction, in particular temporomandibular disorders. ${ }^{(7)}$

Excessive tooth wear is the most frequently cited sign of bruxism. However, it is hazardous to infer that patients have the habit of bruxism from the patterns of wear on their teeth. Although 'bruxofacets' have been defined as atypical facets on teeth, with flat, smooth, shiny areas with sharp edges that correspond with similar opposing areas when the mandible is moved more than $3.5 \mathrm{~mm}$ from centric occlusion in a lateral excursion. ${ }^{(5)}$

Simplistically, the habit of bruxism may produce wear patterns characteristic of attrition on occluding tooth surfaces which are different from the patterns of occlusal tooth-tissue loss associated with dental erosion. Hence, it might be possible to discriminate 'bruxers' from 'nonbruxers' using tooth wear criteria. Conversely, because occlusal wear from bruxism has been found to be frequently associated with cervical erosion, the effects of bruxism on the teeth could be enhanced by enamel demineralization in the acidic environment and hence bruxers would have a higher rate of wear than non-bruxers. ${ }^{(5)}$

Wear facets are suggested to be indicators of these parafunctional habits. They are caused more by long-lasting, dynamic tooth-to-tooth contacts than by other factors (nutrition, salivary buffer) and can be found mainly in the canine and incisor regions. ${ }^{(9)}$
High occlusal wear may become an esthetic problem. Because of the loss of the vertical dimension, the occlusal situation also can be adversely affected. ${ }^{(10,11)}$

Condylar asymmetry, that is the comparison of vertical condylar height $(\mathrm{CH})$ between right and left mandibular condyles, has been used to validate clinical tests of diagnostic categories in patient with TMD. ${ }^{(12)}$

Panoramic radiographs, however, are the most frequently used viewing technique because it is possible to image joints, teeth, and other parts of the jaws in one exposure. Beside mandibular measurements such as tooth length or bone height, panoramic radiographs are now being used as a diagnostic tool in more complicated situations, such as the evaluation of vertical mandibular asymmetry, condylar and ramal height, TMDs, and gonial angle measurements. ${ }^{(13)}$

Aim of this study was to evaluate the effect of teeth wear severity associated with bruxism on the condylar height asymmetry index among different gender using panoramic radiograph viewing technique.

\section{MATERIALS AND METHODS}

Clinical Examination

Selection of the Patients: A total of 150 adult dentate patients who attended outpatient clinic at the College of Dentistry /Mosul University were participate in this study. Participants were divided into two groups 75 bruxers (33 female, 42 male) and 75 non -bruxers control group(39 female, 36 male), age range, 20 35 years and they are reported there is no other oral habits such as gum chewing, nail biting, biting foreign objects, eating seeds and crushing ice. ${ }^{(4)}$ All participants were with normal occlusion met the following criteria ${ }^{(14)}$ :

1.Class I( Angle classification) canine and molar relationship with minor or no crowding; normal growth and development; well aligned upper and lower dental arches.

2. All teeth present.

3. Good facial symmetry determined clinically.

4. No significant medical history. 
5. No history of trauma, or any previous orthodontic, prosthodontic treatment, maxillofacial or plastic surgery.

Impressions and casts of the maxillary and mandibular arches were made.

Assessment of Bruxism:

Bruxism was assessed in the history interview portion of the dental examination. $^{(2,4,5)}$

1.Patients were asked if they exhibited, at least five nights a week, grinding bruxism sounds during sleep during the last 6 months.

2. Observation of tooth wear facets or shiny spots on restorations.

3. Report of morning masticatory muscle fatigue or pain; masseteric hypertrophy upon digital palpation.
3. Presence of marked inner cheek imprint, bilateral prominent linea alba (linea alba on the buccal mucosa, which is cicatricial thickening of the buccal mucosa near the occlusal surfaces of the posterior teeth presumed to be due to tongue thrusting and cheek-biting habits concomitant with the bruxing habit )

4. Presence of indentations on lateral sides of the tongue (impressions of teeth on the tongue and lips).

Measurement of Tooth Wear:

The degree of tooth wear was recorded using the tooth wear index described by Smith and Knight. ${ }^{(15)}$ A score of ( 0 to 4) was given to each of the four surfaces of each tooth ( Table 1),

Table (1): Tooth Wear Index According to Smith and Knight ${ }^{(15)}$

\begin{tabular}{|c|c|c|}
\hline Score* & Surface & Criterion \\
\hline $\mathbf{0}$ & $\begin{array}{l}\mathrm{B} / \mathrm{L} / \mathrm{O} / \mathrm{I} \\
\mathrm{C}\end{array}$ & $\begin{array}{c}\text { No loss of enamel surface characteristics } \\
\text { No change of contour }\end{array}$ \\
\hline 1 & $\begin{array}{l}\mathrm{B} / \mathrm{L} / \mathrm{O} / \mathrm{I} \\
\mathrm{C} \\
\mathrm{B} / \mathrm{L} / \mathrm{O}\end{array}$ & $\begin{array}{l}\text { loss of enamel surface characteristics } \\
\text { Minimal loss of contour } \\
\text { Loss of enamel exposing dentin for less than one third of the surface }\end{array}$ \\
\hline 2 & $\begin{array}{c}\mathbf{I} \\
\mathbf{C} \\
\mathbf{B} / \mathrm{L} / \mathbf{O}\end{array}$ & $\begin{array}{l}\text { Loss of enamel just exposing dentin } \\
\text { Defect less than } 1 \mathrm{~mm} \text { deep } \\
\text { Loss of enamel exposing dentin for more than one third of the surface }\end{array}$ \\
\hline 3 & I & $\begin{array}{l}\text { Loss of enamel and substantial loss of dentin, but not exposing pulp or } \\
\text { secondary dentin }\end{array}$ \\
\hline & $\begin{array}{c}C \\
B / L / O\end{array}$ & $\begin{array}{c}\text { Defect } 1-2 \mathrm{~mm} \text { deep } \\
\text { Complete loss of enamel, pulp exposure, or exposure of secondary dentin }\end{array}$ \\
\hline 4 & $\mathbf{I}$ & $\begin{array}{l}\text { Pulp exposure or exposure of secondary dentin } \\
\text { Defect more than } 2 \mathrm{~mm} \text { deep, pulp exposure, or exposure of secondary } \\
\text { dentin }\end{array}$ \\
\hline
\end{tabular}

and the doubtful cases a lower score was given. The 4 surfaces scored were buccal/ labial, lingual/ palatal, occlusal/ incisal, and the cervical part of the buccal surface. The highest score for each subject was recorded. The severity of tooth wear was graded as grade I mild (score 0 to 1 ) grade II moderate (score 2) and grades III severe (score 3 to 4 ). ${ }^{(16)}$

\section{Radiographic Examination}

Measurement of Condylar Height Asymmetry:

Condylar asymmetry, ramus asymmetry and condylar + ramus asymmetry were determined in the panoramic radiographs by a method developed by Habets et al. ${ }^{(12)}$ (Figure 1). 


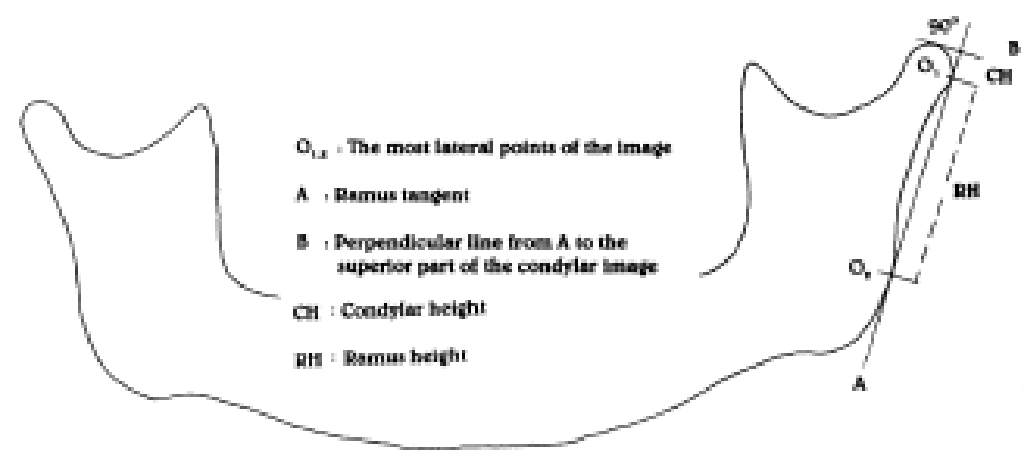

Figure (1): Measuring method according to Habets et $a l^{(12)}$

The panoramic radiographs were taken for each patient using a Panoramic x-ray machine type STRATO 2000, CE by VLLA SISTEMI MEDICALIS- ITALY with regular intensifying screen type Kodak Lanex and $6 \times 12$ inch screen . X-ray machine was operated at $74 \mathrm{kVp}$ and 10 $\mathrm{mA}$. The images after processing were viewed on the viewer. All these radiographs were taken by the same technician in standard manner using the same instrument. The subjects were positioned with the lips in rest position and the head oriented to the Frankfort horizontal plane ${ }^{(14)}$.

All films were traced and measured by the same author. The outlines of the condyle and ascending ramus of both sides on the panoramic radiographs were traced on acetate paper. A line (A) was drawn connecting the most lateral points of the condylar image $\left(\mathrm{O}_{1}\right)$ and the ascending ramus image $\left(\mathrm{O}_{2}\right)$. The distance between $\mathrm{O}_{1}$ and $\mathrm{O}_{2}$ was called the ramus height (RH). To the A line (ramus tangent) from the most superior point of the condylar image, a perpendicular line (B) was drawn. For condylar height $(\mathrm{CH})$, the vertical distance from this line on the $\mathrm{A}$ line to the $\mathrm{O}_{1}$ point was measured (Figure 2) and (Figure3).

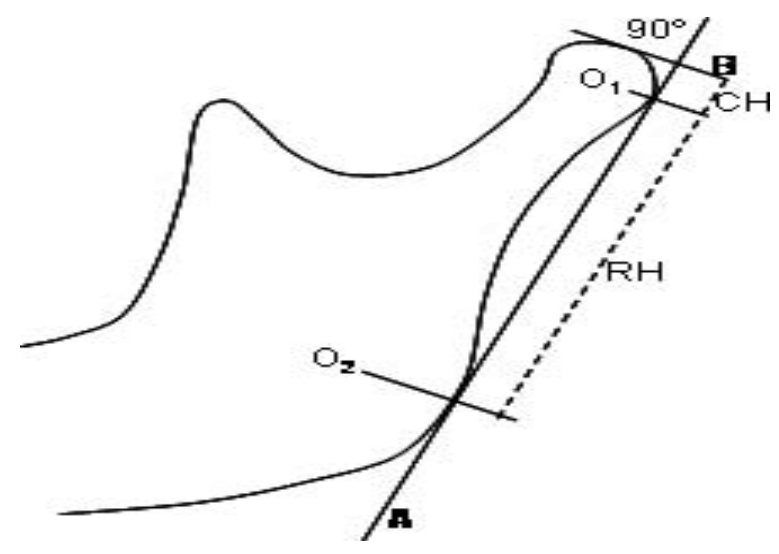

Figure 2. Measuring method according to Habets et al.

$\mathrm{O} 1$ and $\mathrm{O} 2$ indicate the most lateral points of the image; $\mathrm{A}$, ramus tangent; $\mathrm{B}$, perpendicular line from A to the superior part of the condylar image; $\mathrm{CH}$, condylar height; and $\mathrm{RH}$, ramus height. ${ }^{(13)}$ 


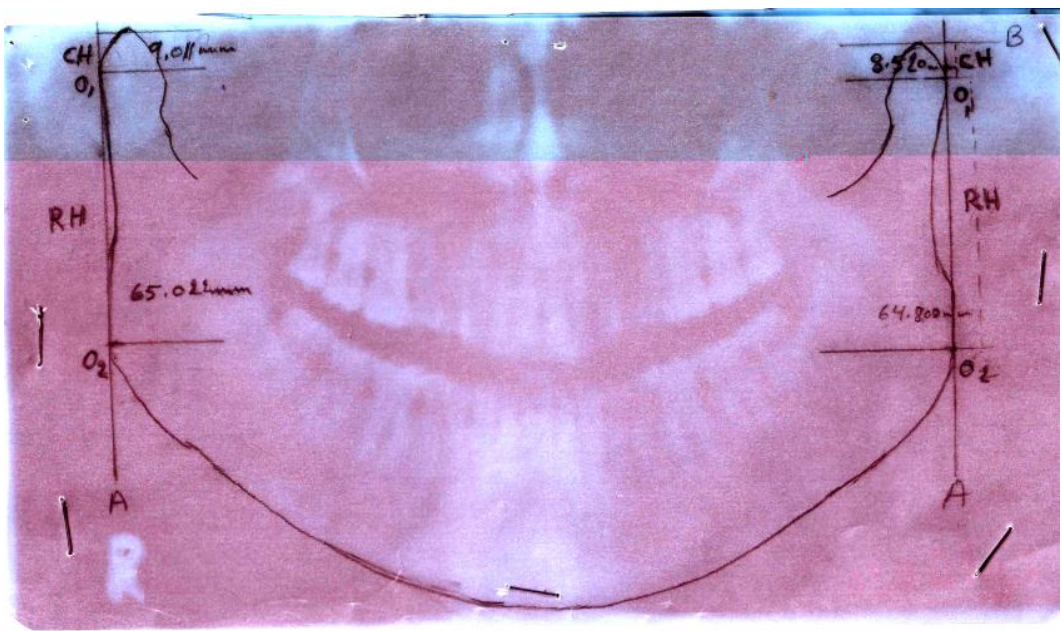

Figure (3): Panoramic image with tracing on acetate tracing paper

Asymmetry index was estimated using the following formula:

Condylar Asymmetry Index: $(\mathrm{CH}$ right $-\mathrm{CH}$ left $/ \mathrm{CH}$ right $+\mathrm{CH}$ left $) \times 100$

Ramus and condyle-plus-ramus asymmetries were also evaluated using the same formula. ${ }^{(13,17,18)}$

All measurements were performed by one investigator on the panoramic radiographs of the subjects using a digital caliper with a $0.01 \mathrm{~mm}$ sensitivity. According to Habets, difference dimensions between both sides of mandible exceeding $3 \%$ suggests asymmetry relation. Habets found that asymmetry index values greater than $3 \%$ must be taken into consideration as vertical asymmetries because of technical errors during film exposure. ${ }^{(14,19)}$ Statistical Analysis

The error of the method was assessed by statistically analyzing the difference between double determinations made 2 weeks apart on the panoramic radiographs of 20 subjects selected at random. A paired sample t-test at the $95 \%$ confidence level showed that the difference between the first and second measurements of the 20 patients was insignificant( Tables 2, 3).

Table (2) : Mean and standard deviation of condylar height measurement of the bruxer subjects among different reading.

\begin{tabular}{cccc}
\hline Group & N & Mean & SD \pm \\
Condylar height measurement $(\mathbf{~ m m})$ & & & \\
\hline First reading & 20 & 7.650 & 1.3774 \\
Second reading & 20 & 7.350 & 1.5313 \\
\hline
\end{tabular}

N: Sample number, SD: Standard Deviation

Table(3): t- Test for the mean of condylar height measurement of the bruxer subjects among different reading..

\begin{tabular}{cccc}
\hline & \multicolumn{3}{c}{ t-test for Equality of Means } \\
\cline { 2 - 4 } Condylar height measurment $(\mathbf{~ m m})$ & $\mathbf{t}$ & $\mathbf{d f}$ & $* \boldsymbol{P}$-value \\
\hline df: degree of freedom, $* P \leq 0.05$ significantly different & 38 & 0.519 \\
\hline
\end{tabular}

All statistical analysis were performed using the SPSS software package (Statistical Package for Social Sciences for Windows, version 10.1). Descriptive statistics and frequencies were computed. Mann-
Whitney U test, Kruskal-Wallis test and Pearson Chi-Square test were used to determine the possible statistically significant differences between groups for vertical mandibular height indices : condylar, 
ramal, and condylar-plus-ramal asymmetry index. Differences between groups were set at $(P \leq 0.05)$.

\section{RESULTS AND DISCUSSION}

The main question regarding the use of panoramic radiographs in evaluating mandibular asymmetries concerns the effect of magnification occurring at the vertical dimensions of the mandible on vertical measurements. Most authors have suggested that small changes in head position do affect horizontal dimensions, while big changes do not occur in vertical dimensions, allowing vertical asymmetry measurements to be performed on panoramic radiographs. Accordingly, panoramic radiographs have been used to compare condylar and ramal heights in different experimental groups, such as denture wearers and patients with TMD or orthodontic anomalies. ${ }^{(13)}$

Means and standard deviation for ver- tical mandibular asymmetry index for bruxer and control (non-bruxer ) subjects are shown in Table(4). The result showed that all asymmetry indices for bruxer group were higher than those of the nonbruxer group which were higher than 3\% (condylar height asymmetry index 5.7656 $\%$, ramal height asymmetry index 3.7160 $\%$, condylar-plus-ramal asymmetry index $3.1532 \%$ ) these indicating asymmetry of the indices. This result come in agreement with the result of Miller et $a l^{(20)}$ in that a group of patients with parafunction showed significantly higher condylar asymmetry than did a group of patients with no noticeable signs of parafunction at $(P \leq 0.05)$. This result explained in that subjects with bruxism have overstrained masticatory muscles leading to hypertrophy and higher bite force ${ }^{(21)}$. Clenching and grinding are parafunctional habits implicated in tempromandibular disorder. ${ }^{(8)}$

Table( 4): Mean and standard deviation for the vertical mandibular height asymmetry indices of the bruxer and non-bruxer subjects.

\begin{tabular}{lllll}
\hline Group & Asymmetry Index \% & N & Mean & SD \pm \\
\hline Bruxism & Condylar height index & 75 & 5.7656 & 5.45766 \\
& Ramus height index & 75 & 3.7160 & 2.30338 \\
& Condylar + Ramus height index & 75 & 3.1532 & 1.33734 \\
Non-Bruxism & 75 & 2.8608 & 2.43604 \\
& Condylar height index & 75 & 1.8920 & 1.60416 \\
& Ramus height index & 75 & 1.4152 & 1.43053 \\
\hline
\end{tabular}

N: Sample number, SD: Standard Deviation

The result in Table (5) showed MannWhitney U Test for the mean of vertical mandibular height asymmetry index. The result showed that there is a statiscally significant difference for all the asymmetry index means between the bruxer and non-bruxer subjects. This result can be explained that the effects of diurnal or nocturnal parafunctional activities can cause destructive effects to dentition, musculature, or joints. ${ }^{(4)}$ Prolonged bruxism may cause intracapsular TMJ derangement, resulting in degenerative disease of that joint. ${ }^{(22)}$ Condylar asymmetry has been related to overloading of the articular surfaces of the tempromandibular joint (TMJ), and it affects the soft and hard tissue components of these tissues, particularly the undifferentiated mesenchymal cell layer. Overloading of the articular surface leads to thickening of the soft tissue component. As a result, there is an increase in the condylar asymmetry which leads to greater muscle hyperactivity, this process can continue until the adaptive capacity of the surface is exhausted, at this point, TMJ problems occur. ${ }^{(12,13)}$ 
Table (5 ) Mann-Whitney U Test for the mean of vertical mandibular height asymmetry indices of the bruxer and non-bruxer subjects.

\begin{tabular}{lccc}
\hline & $\begin{array}{c}\text { Condylar height in- } \\
\text { dex \% }\end{array}$ & $\begin{array}{c}\text { Ramus height in- } \\
\text { dex \% }\end{array}$ & $\begin{array}{c}\text { Condylar + Ramus height } \\
\text { index \% }\end{array}$ \\
\hline Mann- & 17860.500 & 1183.500 & 918.000 \\
Whitney $U$ & -3.945 & -6.137 & -146 \\
$Z$ & 0.000 & 0.000 & 0.000 \\
$*$ p- value & & & \\
\hline$* P \leq 0.05$ significantly different & &
\end{tabular}

Results in Table (6) showed the frequencies and percentage of different gender and tooth wear severity grade among 75 bruxer subjects. The result showed that 42 patients $(56.0 \%)$ were male and 33 patients $(44.0 \%)$ female, and for tooth wear severity grade, 15 patients were mild grade I $(20.0 \%), 42$ patients were grade moderate II $(56.0 \%)$ and 18 patients were severe grade III $(24.0 \%)$.

Table (6): Frequencies for the mean of the condylar height asymmetry index of the bruxer subjects among different gender and tooth wear severity grade.

\begin{tabular}{|c|c|c|c|}
\hline \multicolumn{2}{|c|}{$\begin{array}{l}\text { Condylar height index \% } \\
\text { Group }\end{array}$} & \multirow{2}{*}{$\begin{array}{c}\text { Frequency } \\
42\end{array}$} & \multirow{2}{*}{$\begin{array}{c}\text { Percentage } \% \\
56.0\end{array}$} \\
\hline \multirow{7}{*}{ Tooth wear severity grade } & Male & & \\
\hline & Female & 33 & 44.0 \\
\hline & Total & 75 & 100.0 \\
\hline & Grade I ( mild) & 15 & 20.0 \\
\hline & Grade II (moderate) & 42 & 56.0 \\
\hline & Grade III (severe) & 18 & 24.0 \\
\hline & Total & 75 & 100.0 \\
\hline
\end{tabular}

Results in Table (7) showed the frequencies and percentage of tooth wear severity grade among different genders (75 bruxer subjects). The result showed that tooth wear severity grades for males, 12 patients were mild grade I $(28.6 \%), 24$ patients were grade moderate II $(57.6 \%)$ and 6 patients were severe grade III $(14.3 \%)$. For females 3 patients were mild grade I $(9.1 \%), 18$ patients were grade moderate II $(54.5 \%)$ and 12 patients were severe grade III $(36.4 \%)$.

Table (7) : Frequencies for the mean of the tooth wear severity grades of the bruxer subjects among different genders.

\begin{tabular}{cccc}
\hline Gender & Tooth wear severity grades & Frequency & Percentage\% \\
\hline Males & Grade I & 12 & 28.6 \\
& Grade II & 24 & 57.1 \\
& Grade III & 6 & 14.3 \\
& Total & 42 & 100.0 \\
\hline Females & Grade I & 3 & 9.1 \\
& Grade II & 18 & 54.5 \\
& Grade III & 12 & 36.4 \\
& Total & 33 & 100.0 \\
\hline
\end{tabular}

Results in Table (8) showed Pearson Chi-Square test for the mean of the tooth wear severity grades of the bruxer subjects among different genders . Results showed there is a significant difference between tooth wear among different genders, This result agreed with the result of Pergamalian et $a l^{(1)}$ who found a correlation between tooth wear and different genders, $84 \%$ of the subjects were women with 
higher tooth wear level. But disagreed with the result of Bernhardt et al ${ }^{(10)}$ in that men have higher wear levels than women, which may be explained by stronger masseter muscle function in men.

Table (8): Pearson Chi-Square test for the mean of the tooth wear severity grades of the bruxer subjects among different genders.

\begin{tabular}{lc}
\hline & Tooth wear severity grades \\
\cline { 2 - 2 } Pearson Chi-Square & 7.282 \\
df & 2 \\
$*$ p- value & 0.026 \\
\hline df: degree of freedom $* P \leq 0.05$ significantly different
\end{tabular}

Results in Table (9) showed the mean, standard deviation and Mann- Whitney U test of the condylar asymmetry index of the bruxer subjects among different gender and tooth wear severity grades. The results showed all means of indices were higher than $3 \%$ this indicating asymmetry of the indices. Results also showed that, female have the higher value of condylar asymmetry index $(7.3627 \%)$ than that for male $(4.5107 \%)$. This may be explained in that female hormonal level related to the ovu- latory cycle, has been shown to modulate response to noxious stimuli ${ }^{(23)}$.

The result of Mann- Whitney U test in Table (9) showed that the different between CHI for different gender is statistically insignificant. This result come in agreement with the result of Sezgin etal ${ }^{(13)}$ as the etiology of condylar asymmetries in which sex factor has been investigated and revealed no statistically significant differences.

Table (9) : Mann-Whitney U Test for the mean of condylar height asymmetry index of the bruxer subjects among different genders

\begin{tabular}{lccll}
\hline Condylar height index \% & & & \\
& Gender & N & Mean & SD \pm \\
\cline { 2 - 5 } & Male & 42 & 4.5107 & 3.82556 \\
& Female & 33 & 7.3627 & 6.74032 \\
Mann-Whitney U & 535.500 & & & \\
Z & -1.710 & & & \\
$*_{p \text { - value }}$ & 0.087 & & & \\
\hline
\end{tabular}

$\mathbf{N}$ : Sample number, SD: Standard Deviation* $P \leq 0.05$ significantly different

The result in Table (10) showed means standard deviation and Kruskal-Wallis Test Kruskal-Wallis Test for the condylar height asymmetry index of the bruxer subjects among tooth wear severity grades. The result showed the means for tooth wear severity grades were : grade I (3.7280), grade II (4.9121) and for grade III was the highest value( 10.9700) and all indices means were higher than $3 \%$ that indicating asymmetry of the indices.

The result of Kruskal-Wallis Test for the mean of the CHI of the bruxer subjects in Table (10) showed that there is significant difference between $\mathrm{CHI}$ among tooth wear severity grades. This result is in contrast with the result of Seligman et al ${ }^{(24)}$ in that a relationship between tempromandibular disorders (TMD) and occlusal wear was not found. 
Table (10 ): Kruskal-Wallis Test for the mean of the condylar height asymmetry index of the bruxer subjects among tooth wear severity grades.

\begin{tabular}{lllll}
\hline & \multicolumn{2}{c}{ Condylar height index \% } & & \\
& & & \\
& Tooth wear severity grades & N & Mean & SD \pm \\
& Grade I (mild) & 15 & 3.7280 & 3.68526 \\
& Grade II (moderate) & 42 & 4.9121 & 3.11682 \\
& Grade III (severe) & 18 & 10.9700 & 7.54885 \\
Chi- Squre & & 10.113 & & \\
df & & 2 & & \\
$* \boldsymbol{*}$ - value & & 0.006 & & \\
\hline
\end{tabular}

N: Sample number, SD: Standard Deviation df: degree of freedom , $* P \leq 0.05$ significantly different

\section{CONCLUSIONS}

The result of this study showed that the vertical mandibular asymmetry indices (CHI, RHI and $\mathrm{CH}+\mathrm{RHI}$ )were affected by the tooth wear associated with bruxer subjects, the bruxer subject had the higher value than the non-bruxer subject. Female had higher $\mathrm{CHI}$ than that of the male. Also the result showed that the CHI increased with the tooth wear severity grade increased.

\section{REFERENCES}

1. Pergamalian A, Rudy TE and Zaki HS. The association between wear facets, bruxism, and severity of facial pain in patients with temporomandibular disorders. J Prosthet Dent. 2003;90:194200.

2. Manfredini D, Landi N and Fantoni F. Anxiety symptoms in clinically diagnosed bruxers. J Oral Rehab. 2005 ;32: 584-588

3. Seligman DA and Pullinger AG. Dental attrition models predicting tempromandibular joint disease or masticatory muscle pain versus asymptomatic controls. J Oral Rehab 2006; 33: 789-799

4. Gavish V, Halachimi M and Winocure E. Oral habits and their association with signs and symptoms of tempromandibular disorders in adolescent girls. J Oral Rehab. 2000 ;27: 22-32

5 Khan F, Young WG and Daley TJ. Dental erosion and bruxism. A tooth wear analysis from South East. Queens Austra Den J 1998;43:(2):117-27

6. Lobbezoo F and Naeije M. Review Bruxism is mainly regulated centrally, not peripherally .J Oral Rehab. 2001; 28: 1085-1091
7. Lobbezoo F, Vaderzaag J and Naeije M. Review article in bruxism: its multiple causes and its effects on dental implantsan updated review

J Oral Rehab. 2006; 33: 293-300

8. Ciancaglin R, Gherlone EF and Rdaelli $\mathrm{G}$. The relationship of bruxism with craniofacial pain and symptoms from the masticatory system in the adult population. J Oral Rehab. $2001 ; 28: 842 \pm 848$.

9. Hirsch C, John MT and Labbezoo F. Incisal tooth wear and self-reported TMD Pain in children and adolescents. Int J Prosthodont. 2004; 17:205-210.

10. Bernhardt O, Splieth $C$ and Schwahn C. Risk factors for high occlusal wear scores in a population-based sample: Results of the study of health in pomerania (SHIP). Int J Prosthodont 2004;17:333339.

11. Kevin HK, Smales RJ and John A. Differential wear of teeth and restorative materials: clinical implications. Int $J$ Prosthodont 2004;17:350-356.

12. Shahin AMS The condylar asymmetry measurements in different skeletal patterns J Oral Rehab. 2003;30: 738-742

13. Sezgin OS, Celenk $P$ and Arici S. Mandibular asymmetry in different occlusion patterns. A radiological evaluation. Angle Orthodontist.2007; 77:(5)

14. Kurta GK, Uysal $\mathrm{T}$ and SismanY. Mandibular asymmetry in Class II subdivision malocclusion. Angle Orthodontist. 2008; 78:(1)

15. Al- Hiyasat AS, Khasawneh SF and Khader YS. Tooth wear among psychiatric patients: prevalence, distribution, and associated factors

Int J Prosthodont . 2006;19:403-409.

16. Al-Omiri MK, Lamey PJ and Clifford 
T. Impact of tooth wear on daily living. Int J Prosthodont . 2006;19:601-605.

17. Kurt G, Bayram M and Uysal T. Mandibular asymmetry in cleft lip and palate patients. Eur J Orthod.2009; 0: cjp063v1-cjp063

18. Kurt G. Mandibular asymmetry in cleft lip and palate patients. Eur $J$ Orthod.2010; 32: 19-23 .

19. Syrynska M, Szyszka L and Post M. The use of radiograghs for assessment of asymmetric growth in mandible with AAI. One patient's view report. Pol J Radiol. 2008; 73(3): 17-20 .

20. Miller VJ, Yoeli Z and Barnea E. The effect of parafunction on condylar asymmetry in patients with temporomandibular disorders. J Oral Rehab. 1998; 25: 721-724.

21. Cosme DC, Baldisseotto SM and

Canabarro SA. Bruxism and voluntary maximal bite force in young dentate adult. Int J Prosthodont 2005;18:328332.

22. Shen YH, Chen YK and Chang SY. Condylar resorption during active orthodontic treatment and subsequent therapy: report of a special case dealing with iatrogenic TMD possibly related to orthodontic treatment. J Oral Rehab. 2005; 32: 332-336.

23. Plesh O, Curtis DA and Hall LJ.Gender difference in jaw pain induced by clenching. I Oral Rehab. 1998; 25: 258-263.

24.Seligman DA, Pullinger AG and Solberg WK. The prevalence of dental attrition and its association with factors of age, gender, occlusion, and TMJ symptomatology. J Dent Res. 1988;67:13231333. 